\title{
Nonsmoothable group actions on spin 4-manifolds
}

\author{
KAZUHIKO KIYONO
}

\begin{abstract}
We show that every closed, simply connected, spin topological 4-manifold except $S^{4}$ and $S^{2} \times S^{2}$ admits a homologically trivial, pseudofree, locally linear action of $\mathbb{Z}_{p}$ for any sufficiently large prime number $p$ which is nonsmoothable for any possible smooth structure.
\end{abstract}

57M60; 57R57

\section{Introduction}

In this article we call a locally linear action of a group on a topological manifold nonsmoothable if the action is not smooth with respect to any possible smooth structure. Several authors have been investigated examples of nonsmoothable group actions on 4-manifolds. See Kwasik and Lee [14], Kwasik and Lawson [13], Hambleton and Lee [9], Bryan [1], Hambleton and Tanase [10] and Nakamura [17].

We restrict our attention to actions of the cyclic groups of odd prime order which are homologically trivial and pseudofree. A L Edmonds constructed such actions on all simply connected 4-manifolds [6]. The main purpose of this article is to show that there is a family of locally linear actions constructed by Edmonds's method which are nonsmoothable.

Theorem 1.1 Let $X$ be a closed, simply connected, spin topological 4-manifold not homeomorphic to either $S^{4}$ or $S^{2} \times S^{2}$. Then, for any sufficiently large prime number $p$, there exists a homologically trivial, pseudofree, locally linear action of $\mathbb{Z}_{p}$ on $X$ which is nonsmoothable.

The conclusion of Theorem 1.1 does not hold for $S^{4}$. It is known that every pseudofree locally linear action of odd order cyclic group on $S^{4}$ is smooth with respect to a smooth structure isomorphic to the standard one (see Wilczyński [18]). Concerning smooth actions on $S^{2} \times S^{2}$, M Klemm obtained partial results [11], while the following problem seems open.

Problem 1.2 Is there a homologically trivial, pseudofree, nonsmoothable locally linear action of $\mathbb{Z}_{p}$ on $S^{2} \times S^{2}$ for some odd prime number $p$ ? 
Let NS $(X)$ denote the set of all prime numbers $p$ for which $X$ admits a homologically trivial, pseudofree, nonsmoothable locally linear action of $\mathbb{Z}_{p}$.

Theorem 1.3 For any closed, simply connected, spin 4-manifold $X$ not homeomorphic to $S^{4}$ or $S^{2} \times S^{2}, \mathrm{NS}(X)$ contains all the prime numbers $p$ satisfying

$$
p \geq 12\left\lceil\frac{\max \left\{b_{2}^{+}(X), b_{2}^{-}(X)\right\}+1}{2}\right\rceil-5 .
$$

Here $\lceil x\rceil$ is the maximum integer less than or equal to $x$. Though we need to fix an orientation of $X$ to define $b_{2}^{+}(X)$ and $b_{2}^{-}(X)$, the right-hand side of the above estimate of $p$ does not depend on the choice.

The above estimate is not best possible. We show a better estimate for the connected sums of the copies of $S^{2} \times S^{2}$.

Theorem 1.4 (1) $\mathrm{NS}\left(\left(S^{2} \times S^{2}\right) \#\left(S^{2} \times S^{2}\right)\right)$ contains all the prime numbers $p \geq 7$.

(2) For $n \geq 3$, NS(\# $\left.\#^{n} S^{2} \times S^{2}\right)$ contains all the prime numbers $p \geq 19$.

We also obtain the following.

\section{Theorem $1.511 \in \mathrm{NS}(K 3)$.}

We prove Theorem 1.1 in three steps. In Section 2 we give a family of homologically trivial, pseudofree, locally linear actions, slightly modifying the construction of Edmonds in [6] and making use of the criterion of Edmonds and J H Ewing in [7]. In Section 3 we calculate the dimension of $\mathbb{Z}_{p}$-invariant part of the $\mathbb{Z}_{p}$-index of the Dirac operator for the action constructed in Section 2, assuming that $X$ is spin and that the action is smooth for some smooth structure (Proposition 3.3). The dimension is equal to the index of the Dirac operator on the quotient $V$-manifold $X / \mathbb{Z}_{p}$. In Section 4 we prove Theorem 1.1 applying the 10/8-type inequality for the quotient $V$-manifold $X / \mathbb{Z}_{p}$ in Fukumoto and Furuta [8].

Remark 1.6 Presumably the estimate in Theorem 1.4 could be improved further in general. We also do not know the set $\mathrm{NS}(K 3)$ exactly while Theorem 1.3 tells that $\mathrm{NS}(K 3)$ contains all the prime numbers greater than 113 .

Remark 1.7 When a smooth structure is given on a topological manifold, a locally linear group action on the topological manifold is called nonsmoothable if the action is not smooth with respect to any smooth structure equivalent to the given one. W Chen and S Kwasik constructed group actions on $K 3$ surface of this type, which are smooth 
with respect to the standard smooth structure but not smooth with respect to infinitely many exotic structures [2]. X Liu and N Nakamura constructed group actions on elliptic surfaces which are not smooth with respect to infinitely many smooth structures including the standard smooth structure [15; 16]. It is not known whether the examples of Liu and Nakamura are nonsmoothable for every smooth structure or not. Liu and Nakamura used mod $p$ vanishing theorem of Seiberg-Witten invariant for 4-manifolds with nonvanishing Seiberg-Witten invariant. Recently Nakamura applied a similar method to $K 3$ \# K3, for which the Seiberg-Witten invariant is zero but its cohomotopy refinement does not vanish [17]. We also use Seiberg-Witten theory to investigate nonsmoothability of finite group action. Our approach is to apply an equivariant version of 10/8-inequality to spin 4-manifolds, which does not depend on nonvanishing of Seiberg-Witten invariant.

Acknowledgements The author thanks Mikio Furuta for his invaluable advice and encouragement, Allan Edmonds for useful information on pseudofree, locally linear group actions on $S^{4}$, and Yukio Kametani and Nobuhiro Nakamura for helpful discussion.

\section{Locally linear actions}

Let $X$ be a closed, oriented, simply connected topological 4-manifold not necessarily spin. Edmonds proved the following theorem.

Theorem 2.1 (Edmonds [6, Theorem 6.4]) For any prime number $p$ not less than 5 there exists a homologically trivial, pseudofree, locally linear action of $\mathbb{Z}_{p}$ on $X$.

Edmonds constructed the group action using equivariant surgery on the connected sum of $b_{2}^{+}(X)$ copies of $\mathbb{C} P^{2}$ and $b_{2}^{-}(X)$ copies of $\overline{\mathbb{C} P^{2}}$ for some choice of $\mathbb{Z}_{p^{-}}$ action. Moreover Edmonds and Ewing obtained a necessary and sufficient criterion for realizability of a pair of a fixed point data and a unimodular quadratic form with $\mathbb{Z}_{p}$-action by a pseudofree locally linear $\mathbb{Z}_{p}$-action on $X$ [7]. In this section we follow Edmonds's construction with a slight modification to obtain a family of fixed point data satisfying Edmonds and Ewing's criterion. More specifically, we make realizable fixed point data by gathering the fixed point data of pseudofree $\mathbb{Z}_{p}$-actions on $\mathbb{C} P^{2}$, $\overline{\mathbb{C} P^{2}}$ and $S^{4}$.

We identify $\mathbb{Z}_{p}$ with the subgroup of $U(1)$ and, for an integer $a$, let $\mathbb{C}_{a}$ be the one-dimensional complex representation of $\mathbb{Z}_{p}$ defined by $z \mapsto g^{a} z$ for $z \in \mathbb{C}$ and $g \in \mathbb{Z}_{p}$. 
Definition 2.2 Using weights $\alpha=\left(a_{0}, a_{1}, a_{2}\right), \alpha^{\prime}=\left(a_{0}^{\prime}, a_{1}^{\prime}, a_{2}^{\prime}\right)$ and $\beta=\left(b_{1}, b_{2}\right)$ respectively define $\mathbb{Z}_{p}$-manifolds $\mathbb{C} P_{\alpha}^{2}, \overline{\mathbb{C} P_{\alpha^{\prime}}^{2}}$ and $S_{\beta}^{4}$ as $\mathbb{C} P^{2}, \overline{\mathbb{C} P^{2}}$ and $S^{4}$ with pseudofree $\mathbb{Z}_{p}$-actions as follows.

(1) Suppose $a_{0}, a_{1}$ and $a_{2}$ are integers which are not congruent modulo $p$ to each other. Let $\mathbb{C} P_{\alpha}^{2}$ denote the quotient space $\left(\mathbb{C}_{a_{0}} \oplus \mathbb{C}_{a_{1}} \oplus \mathbb{C}_{a_{2}} \backslash\{(0,0,0)\}\right) / \mathbb{C}^{*}$.

(2) Suppose $a_{0}^{\prime}, a_{1}^{\prime}$ and $a_{2}^{\prime}$ are integers which are not congruent modulo $p$ to each other. Denote $\mathbb{C} P_{\alpha^{\prime}}^{2}$ with the opposite orientation by $\overline{\mathbb{C} P_{\alpha^{\prime}}^{2}}$.

(3) Suppose $b_{1}$ and $b_{2}$ are integers not congruent to 0 modulo $p$. Let $S_{\beta}^{4}$ denote the unit sphere of $\mathbb{C}_{b_{1}} \oplus \mathbb{C}_{b_{2}} \oplus \mathbb{R}$, where $\mathbb{R}$ is the trivial one-dimensional real representation of $\mathbb{Z}_{p}$.

Note that the two weights $\alpha_{0}=\left(a_{0}, a_{1}, a_{2}\right)$ and $\alpha_{1}=\left(a_{0}+1, a_{1}+1, a_{2}+1\right)$ give the same action on $\mathbb{C} P^{2}$ hence $\mathbb{C} P_{\alpha_{0}}^{2}$ and $\mathbb{C} P_{\alpha_{1}}^{2}$ are $\mathbb{Z}_{p}$-equivariantly diffeomorphic. From now on we assume that $a_{0}+a_{1}+a_{2}$ and $a_{0}^{\prime}+a_{1}^{\prime}+a_{2}^{\prime}$ are even for weights $\alpha=\left(a_{0}, a_{1}, a_{2}\right)$ and $\alpha^{\prime}=\left(a_{0}^{\prime}, a_{1}^{\prime}, a_{2}^{\prime}\right)$ in Definition 2.2 without loss of generality.

To make realizable fixed point data from the data coming from $\mathbb{C} P_{\alpha}^{2}$ 's, $\overline{\mathbb{C} P_{\alpha^{\prime}}^{2}}$, s and $S_{\beta}^{4}$ 's we may need to reduce the number of fixed points by removing certain pairs as in the following definition.

Definition 2.3 We call a pair of fixed points a cancelling pair if there is a weight $\beta$ such that the fixed point data of $S_{\beta}^{4}$ coincides with that of the pair. We also call such a weight $\beta$ a weight of the cancelling pair.

A pair of fixed points is a cancelling pair if and only if the two isotropy representations at the two fixed points are isomorphic to each other through an orientation-reversing isomorphism. The weight of the cancelling pair is one of the weights of these representations. (We have two possible representatives of weights for each cancelling pair.)

We will use the following cancelling pairs later. These examples are special cases of Edmonds [6, Lemma 6.2].

Example 2.4 Let $p$ be a prime number not less than 5 .

(1) Let $a, b$ and $c$ be integers satisfying $a-b, a-b-c, a-b-2 c, c \not \equiv 0 \bmod p$ and $a \equiv c \bmod 2$. For $\alpha_{1}=(a, b, b+c)$ and $\alpha_{2}=(a, b+c, b+2 c)$ the pair $[0,0,1]$ on $\mathbb{C} P_{\alpha_{1}}^{2}$ and $[0,1,0]$ on $\mathbb{C} P_{\alpha_{2}}^{2}$ is a cancelling pair. 
(2) Let $i$ be an integer satisfying $i \not \equiv-1,-2,-3 \bmod p$. For $\alpha_{1}=(-1, i, i+1)$ and $\alpha_{2}=(-1, i+1, i+2)$ the pair of $[0,0,1]$ on $\mathbb{C} P_{\alpha_{1}}^{2}$ and $[0,1,0]$ on $\mathbb{C} P_{\alpha_{2}}^{2}$ is a cancelling pair.

(3) For $\alpha_{1}=(-1, p-4, p-3)$ and $\alpha_{2}=(-1,0,1)$ the pair of $[0,0,1]$ on $\mathbb{C} P_{\alpha_{1}}^{2}$ and $[1,0,0]$ on $\mathbb{C} P_{\alpha_{2}}^{2}$ is a cancelling pair.

(4) Let $n$ be a positive integer. For each $1 \leq i \leq n$ we write $R(i)$ for the remainder of $i$ divided by $p-3$ and $\alpha_{i}$ for the weight $(-1, R(i), R(i)+1)$. There are $n-1$ cancelling pairs in the fixed points of the disjoint union $\bigsqcup_{1 \leq i \leq n} \mathbb{C} P_{\alpha_{i}}^{2}$.

Note that (2) is the special case of (1) with $a=-1, b=i$ and $c=1,(3)$ is essentially the case (2) with $i=p-4$ since weights $(-1,0,1)$ and $(p-3, p-2, p-1)$ induce the same action, and (4) is a consequence of the cases (2) and (3).

Proposition 2.5 Let $X$ be a closed, oriented, simply connected topological 4-manifold, and $m, m^{\prime}, r$ and $s$ nonnegative integers satisfying

$$
m-m^{\prime}=\sigma(X) \quad \text { and } \quad 3\left(m+m^{\prime}\right)+2(r+s)=\chi(X),
$$

where $\sigma(X)$ and $\chi(X)$ are the signature and the Euler number of $X$ respectively. Suppose there are weights $\alpha_{i}(1 \leq i \leq m), \alpha_{j}^{\prime}\left(1 \leq j \leq m^{\prime}\right)$ and $\beta_{k}(1 \leq k \leq r)$ such that the fixed point set of the disjoint union

$$
\left(\coprod_{1 \leq i \leq m} \mathbb{C} P_{\alpha_{i}}^{2}\right) \sqcup\left(\coprod_{1 \leq j \leq m^{\prime}} \overline{\mathbb{C} P_{\alpha_{j}^{\prime}}^{2}}\right) \sqcup\left(\coprod_{1 \leq k \leq r} S_{\beta_{k}}^{4}\right)
$$

has $s$ cancelling pairs. Let $\mathcal{D}$ be the fixed point data for those fixed points which does not appear in the $s$ cancelling pairs. Then there exists a homologically trivial, pseudofree, locally linear action of $\mathbb{Z}_{p}$ on $X$ whose fixed point data is the same as $\mathcal{D}$.

Proof We check that the data $\mathcal{D}$ satisfies the three conditions REP, GSF and TOR in Edmonds and Ewing [7]. We write $Y$ for the disjoint union (2-1). Let $\gamma_{l} \quad(1 \leq l \leq s)$ be weights of the $s$ cancelling pairs on $Y$ and we write $Z$ for the disjoint union:

$$
Z=\coprod_{1 \leq l \leq s} S_{\gamma_{l}}^{4} \text {. }
$$

Since the number of fixed points of $\mathcal{D}$ is $3 m+3 m^{\prime}+2 r-2 s=\chi(X), \mathcal{D}$ satisfies the condition REP for homologically trivial action on $X$.

The right-hand side of GSF for $\mathcal{D}$ is the difference between those for the fixed point data of $Y$ and of $Z$. This is equal to $\sigma(Y)-\sigma(Z)$ since the condition GSF is true for 
both $Y$ and $Z$. By the assumption of the proposition, $\sigma(Y)-\sigma(Z)=m-m^{\prime}=\sigma(X)$. So $\mathcal{D}$ satisfies also the condition GSF for homologically trivial action on $X$.

The condition TOR is equivalent to the equation of [7, Application 8.6] for homologically trivial action. Let $L(\mathcal{D}), L(Y)$ and $L(Z)$ be the left-hand sides of the equations for $\mathcal{D}$, for the fixed point data of $Y$ and for that of $Z$ respectively. Note that we have $L(\mathcal{D})=L(Y) / L(Z)$. Since the fixed point data of $Y$ and that of $Z$ satisfy

$$
L(Y) \approx(-1)^{m} \times(-(-1))^{m^{\prime}} \times(-1)^{r}=(-1)^{m+r} \quad \text { and } \quad L(Z) \approx(-1)^{s},
$$

we obtain

$$
L(\mathcal{D}) \approx(-1)^{m+r-s}=(-1)^{b_{2}^{-}(X)+1} .
$$

This is the equation for $\mathcal{D}$.

Edmonds used the construction with $\left(m, m^{\prime}, r, s\right)=\left(b_{2}^{+}(X), b_{2}^{-}(X), 0, b_{2}(X)-1\right)$ to prove Theorem 2.1 in [6]. We will use other choices of $\left(m, m^{\prime}, r, s\right)$ to prove Theorem 1.1, Theorem 1.4 and Theorem 1.5.

\section{Index of Dirac operator}

In this section we calculate the dimension of $\mathbb{Z}_{p}$-invariant part of the $\mathbb{Z}_{p}$-index of the Dirac operator on $X$ for the $\mathbb{Z}_{p}$-action given in Proposition 2.5, assuming that $X$ is a spin smooth manifold and that the $\mathbb{Z}_{p}$-action is smooth. We use in the calculation the differential operator on the disjoint manifold (2-1) whose restriction on any component homeomorphic to $\mathbb{C} P^{2}$ is the twisted Dolbeault operator defined in Lemma 3.2 below.

Recall that we are assuming that $a_{0}+a_{1}+a_{2}$ is even for a weight $\alpha=\left(a_{0}, a_{1}, a_{2}\right)$. Let $|\alpha|$ be $a_{0}+a_{1}+a_{2}$.

Definition 3.1 Define a nonnegative integer $N(p, \alpha)$ as the number of ordered triplets of integer $\left(n_{0}, n_{1}, n_{2}\right)$ satisfying

$$
n_{0}, n_{1}, n_{2} \geq 0, \quad n_{0}+n_{1}+n_{2}=\frac{p-3}{2}, \quad a_{0} n_{0}+a_{1} n_{1}+a_{2} n_{2}+\frac{|\alpha|}{2} \equiv 0 \bmod p .
$$

Lemma 3.2 The dimension of $\mathbb{Z}_{p}$-invariant part of the $\mathbb{Z}_{p}$-index of the Dolbeault operator on $\mathbb{C} P_{\alpha}^{2}$ with coefficient $\mathcal{O}((p-3) / 2) \otimes \mathbb{C}_{-|\alpha| / 2}$ is equal to $N(p, \alpha)$.

Proof Let $L_{\alpha}$ be the $\mathbb{Z}_{p}$-equivariant line bundle $\mathcal{O}((p-3) / 2) \otimes \mathbb{C}_{-|\alpha| / 2}$ and $\bar{\partial}_{\alpha}$ the twisted Dolbeault operator on $\mathbb{C} P_{\alpha}^{2}$ with coefficient $L_{\alpha}$. We have by Dolbeault's 
theorem [3] the following equality between virtual representations, where the left-hand side is the $\mathbb{Z}_{p}$-index of the operator $\bar{\partial}_{\alpha}$ :

$$
\operatorname{ind}_{\mathbb{Z}_{p}} \bar{\partial}_{\alpha}=H^{0}\left(\mathbb{C} P^{2} ; \mathcal{O}\left(L_{\alpha}\right)\right)-H^{1}\left(\mathbb{C} P^{2} ; \mathcal{O}\left(L_{\alpha}\right)\right)+H^{1}\left(\mathbb{C} P^{2} ; \mathcal{O}\left(L_{\alpha}\right)\right)
$$

Since $\mathbb{C} P^{2}$ is Kähler and the holomorphic line bundle $K^{-1} \otimes L_{\alpha}$ is positive, where $K$ is the canonical line bundle of $\mathbb{C} P^{2}$, both $H^{1}\left(\mathbb{C} P^{2} ; \mathcal{O}\left(L_{\alpha}\right)\right)$ and $H^{2}\left(\mathbb{C} P^{2} ; \mathcal{O}\left(L_{\alpha}\right)\right)$ are zero because of Kodaira's vanishing theorem [12]. The $\mathbb{Z}_{p}$-index ind $\mathbb{Z}_{p} \bar{\partial}_{\alpha}$ is hence equal to the actual representation $H^{0}\left(\mathbb{C} P^{2} ; \mathcal{O}\left(L_{\alpha}\right)\right)$, which is

$$
\operatorname{Span}\left\{z_{0}^{n_{0}} z_{1}^{n_{1}} z_{2}^{n_{2}} \mid n_{0}, n_{1}, n_{2} \geq 0, n_{0}+n_{1}+n_{2}=\frac{p-3}{2}\right\}
$$

with $\mathbb{Z}_{p}$-action

$$
\begin{aligned}
& g\left[z_{0}, z_{1}, z_{2} ; z_{0}^{n_{0}} z_{1}^{n_{1}} z_{2}^{n_{2}}\right]=\left[g^{a_{0}} z_{0}, g^{a_{1}} z_{1}, g^{a_{2}} z_{2} ; g^{-|\alpha| / 2} z_{0}^{n_{0}} z_{1}^{n_{1}} z_{2}^{n_{2}}\right] \\
& =\left[g^{a_{0}} z_{0}, g^{a_{1}} z_{1}, g^{a_{2}} z_{2} ; g^{-\sum a_{i} n_{i}-|\alpha| / 2}\left(g^{a_{0}} z_{0}\right)^{n_{0}}\left(g^{a_{1}} z_{1}\right)^{n_{1}}\left(g^{n_{2}} z_{2}\right)^{n_{2}}\right] .
\end{aligned}
$$

This completes the proof.

Proposition 3.3 Let $X$ be a closed, simply connected, spin smooth 4-manifold. Suppose a $\mathbb{Z}_{p}$-action constructed in Proposition 2.5 is smooth with respect to some smooth structure of $X$. Then the action has the unique lift to the spin structure on $X$ and the dimension of $\mathbb{Z}_{p}$-invariant part of the $\mathbb{Z}_{p}$-index of the Dirac operator on $X$ is equal to:

$$
\sum_{i=1}^{m} N\left(p, \alpha_{i}\right)-\sum_{j=1}^{m^{\prime}} N\left(p, \alpha_{j}^{\prime}\right)-\frac{\sigma(X)}{8} p
$$

Proof Let $Y$ be the disjoint union of $\mathbb{C} P_{\alpha_{i}}^{2}$ 's, $\overline{\mathbb{C} P_{\alpha_{j}^{\prime}}^{2}}$ 's and $S_{\beta_{k}}^{4}$ 's of (2-1) in Proposition 2.5, $Z$ the disjoint union of $S_{\gamma_{l}}^{4}$ 's of (2-2) in the proof of it, and $X^{\prime}$ the disjoint union of $X$ and $Z$. Note that $Z$ and $X^{\prime}$ are spin. In general, since $p$ is odd, $\mathbb{Z}_{p}$-actions on spin manifolds have unique lift to spin structures. Let $D_{X}, D_{Z}$ and $D_{X^{\prime}}$ be the Dirac operators on $X, Z$ and $X^{\prime}$ respectively. Since $\operatorname{ind}_{\mathbb{Z}_{p}} D_{Z}=0$ we have $\operatorname{ind}_{\mathbb{Z}_{p}} D_{X^{\prime}}=\operatorname{ind}_{\mathbb{Z}_{p}} D_{X}$. We construct below a $\mathbb{Z}_{p}$-equivariant $\operatorname{spin}^{c}$ structure on $Y$ so that it is $\mathbb{Z}_{p}$-equivariantly spin on a neighborhood of the fixed point set, and compare $\operatorname{ind}_{\mathbb{Z}_{p}} D_{X^{\prime}}$ with the $\mathbb{Z}_{p}$-index of the $\operatorname{spin}^{c}$ Dirac operator of it.

The $\mathbb{Z}_{p}$-equivariant $\operatorname{spin}^{c}$ structure on each $\mathbb{C} P_{\alpha_{i}}^{2}$-component of $Y$ is defined so that its $\operatorname{spin}^{c}$ Dirac operator is identified with the Dolbeault operator twisted by $L_{\alpha_{i}}$ in Lemma 3.2. The square of $L_{\alpha_{i}}$ is $\mathbb{Z}_{p}$-equivariantly isomorphic to $K \otimes \mathcal{O}(p)$, where $K$ is the canonical line bundle of $\mathbb{C} P^{2}$. Since $\mathcal{O}(p)$ is $\mathbb{Z}_{p}$-equivariantly trivial on a 
neighborhood of the fixed point set, $L_{\alpha_{i}}$ is a $\mathbb{Z}_{p}$-equivariant square root of $K$ there. It implies that the $\operatorname{spin}^{c}$ structure is $\mathbb{Z}_{p}$-equivariantly spin on a neighborhood of the fixed point set. The $\mathbb{Z}_{p}$-equivariant $\operatorname{spin}^{c}$ structure on each $\overline{\mathbb{C} P_{\alpha_{j}^{\prime}}^{2}}$-component of $Y$ is defined so that the $\operatorname{spin}^{c}$ Dirac operator is identified with the same twisted Dolbeault operator with opposite parity of degree. The $\mathbb{Z}_{p}$-equivariant $\operatorname{spin}^{c}$ structure on each $S_{\beta_{k}}^{4}$-component of $Y$ is defined as $\mathbb{Z}_{p}$-equivariant spin structure.

Let $D_{Y}$ be the $\operatorname{spin}^{c}$ Dirac operator on the $\operatorname{spin}^{c}$ structure defined as above. Since the spin action on $X^{\prime}$ is isomorphic to the $\operatorname{spin}^{c}$ action on $Y$ on neighborhoods of their fixed point sets ind $g D_{X^{\prime}}$ and $\operatorname{ind}_{g} D_{Y}$ coincide for $g \neq 1 \in \mathbb{Z}_{p}$. This is a consequence of the localization of the equivariant indices as elements of some localization of equivariant $K$-groups of the neighborhoods of the fixed point sets, or one could also see it from the Atiyah-Segal-Lefschetz formula. Hence we first obtain

$$
\begin{aligned}
& \operatorname{dim}\left(\operatorname{ind}_{\mathbb{Z}_{p}} D_{X^{\prime}}\right)^{\mathbb{Z}_{p}}-\operatorname{dim}\left(\operatorname{ind}_{\mathbb{Z}_{p}} D_{Y}\right)^{\mathbb{Z}_{p}}=\frac{1}{p} \sum_{g \in \mathbb{Z}_{p}} \operatorname{ind}_{g} D_{X^{\prime}}-\frac{1}{p} \sum_{g \in \mathbb{Z}_{p}} \operatorname{ind}_{g} D_{Y} \\
& =\frac{1}{p}\left(\operatorname{ind}_{1} D_{X^{\prime}}-\operatorname{ind}_{1} D_{Y}\right) \\
& =\frac{1}{p}\left(\text { ind } D_{X^{\prime}}-\text { ind } D_{Y}\right) \text {. }
\end{aligned}
$$

Secondly, from the Hirzebruch signature theorem and a direct calculation, we have

$$
\begin{aligned}
& \text { ind } D_{X^{\prime}}=-\frac{\sigma\left(X^{\prime}\right)}{8}=-\frac{\sigma(X)}{8} \\
& \text { ind } D_{Y}=\left(m-m^{\prime}\right) \operatorname{dim} H^{0}\left(\mathbb{C} P^{2} ; \mathcal{O}((p-3) / 2)\right)=\sigma(X) \frac{p^{2}-1}{8}
\end{aligned}
$$

which imply

$$
\frac{1}{p}\left(\text { ind } D_{X^{\prime}}-\text { ind } D_{Y}\right)=-\sigma(X) \frac{p}{8} \text {. }
$$

Thirdly, applying Lemma 3.2 to each component of $Y$, we have

$$
\operatorname{dim}\left(\operatorname{ind}_{\mathbb{Z}_{p}} D_{Y}\right)^{\mathbb{Z}_{p}}=\sum_{i=1}^{m} N\left(p, \alpha_{i}\right)-\sum_{j=1}^{m^{\prime}} N\left(p, \alpha_{j}^{\prime}\right) .
$$

Now Equations (3-2), (3-3) and (3-4) imply the required formula.

Remark 3.4 The dimension of $\mathbb{Z}_{p}$-invariant part of the $\mathbb{Z}_{p}$-index of the Dirac operator on $X$ is nothing but the index of the Dirac operator on the quotient spin $V$-manifold $X / \mathbb{Z}_{p}$. 


\section{Nonsmoothability}

In this section we prove Theorem 1.1 choosing appropriate weights and using the 10/8-type inequality for the quotient $V$-manifold $X / \mathbb{Z}_{p}$ in Fukumoto and Furuta [8].

Theorem 4.1 Let $X$ be a closed, simply connected, spin smooth 4-manifold not homeomorphic to $S^{4}$. Suppose the integers $m, m^{\prime}, r, s$ and $\mathbb{Z}_{p}$-manifolds $\mathbb{C} P_{\alpha_{i}}^{2}$ $(1 \leq i \leq m), \overline{\mathbb{C} P_{\alpha_{j}^{\prime}}^{2}}\left(1 \leq j \leq m^{\prime}\right), S_{\beta_{k}}^{4}(1 \leq k \leq r)$ satisfy the assumption of Proposition 2.5. If the $\mathbb{Z}_{p}$-action on $X$ constructed in Proposition 2.5 is smooth with respect to some smooth structure of $X$, then we have the inequality

$$
-b_{2}^{-}(X)<\sum_{i=1}^{m} N\left(p, \alpha_{i}\right)-\sum_{j=1}^{m^{\prime}} N\left(p, \alpha_{j}^{\prime}\right)-\frac{\sigma(X)}{8} p<b_{2}^{+}(X) .
$$

Proof In general when a finite group $G$ acts on a closed, spin smooth 4-manifold $W$ preserving its orientation and the spin structure, Y Fukumoto and M Furuta showed the inequality

$$
\operatorname{dim}\left(\operatorname{ind}_{G} D\right)^{G}<\operatorname{dim}_{\mathbb{R}} H_{+}^{2}(W ; \mathbb{R})^{G}
$$

when the right-hand side is not zero, where $D$ is the $G$-equivariant Dirac operator on $W$ [8]. In our case, since the action is homologically trivial, the right-hand side for $W=X$ is equal to $b_{2}^{+}(X)$. When $X$ is a spin smooth manifold not homeomorphic to $S^{4}$ a theorem of S K Donaldson implies $b_{2}^{+}(X), b_{2}^{-}(X)>0$ [4]. Therefore if the action is smoothable we have the above inequality. Using the formula given by Proposition 3.3 we can write the inequality as

$$
\sum_{i=1}^{m} N\left(p, \alpha_{i}\right)-\sum_{j=1}^{m^{\prime}} N\left(p, \alpha_{j}^{\prime}\right)-\frac{\sigma(X)}{8} p<b_{2}^{+}(X) .
$$

Reversing the orientation of $X$ we similarly obtain

$$
\sum_{j=1}^{m^{\prime}} N\left(p, \alpha_{j}^{\prime}\right)-\sum_{i=1}^{m} N\left(p, \alpha_{i}\right)+\frac{\sigma(X)}{8} p<b_{2}^{-}(X) .
$$

Proofs of Theorems 1.1 and 1.3 When $X$ has no smooth structure Theorem 1.1 is included in Edmonds's Theorem 2.1. So we assume below that $X$ has a smooth structure. We also assume $\sigma(X) \leq 0$ giving the opposite orientation to $X$ if necessary.

To construct an action which does not satisfy the inequality of Theorem 4.1 we choose different four-tuple $\left(m, m^{\prime}, r, s\right)$ from that used by Edmonds in [6]. 
Lemma 4.2 Let $p$ be a prime number not less than 5 and $X$ a closed, simply connected, spin smooth 4-manifold with $\sigma(X) \leq 0$ not homeomorphic to $S^{4}$ or $S^{2} \times S^{2}$. Then there exist weights

$$
\alpha_{1}^{\prime}, \alpha_{2}^{\prime}, \ldots, \alpha_{-\sigma(X)}^{\prime}
$$

for $\overline{\mathbb{C} P^{2}}$ satisfying the following property: For any weights $\alpha_{0}$ and $\alpha_{0}^{\prime}$, the $\mathbb{Z}_{p}-$ manifolds $\mathbb{C} P_{\alpha_{0}}^{2}, \overline{\mathbb{C} P_{\alpha_{j}^{\prime}}^{2}}(0 \leq j \leq-\sigma(X))$ and $S_{\beta_{k}}^{4}(1 \leq k \leq r)$ for some nonnegative integer $r$ and some weights $\beta_{k}(1 \leq k \leq r)$ satisfy the assumption of Proposition 2.5.

Proof For each $1 \leq j \leq-\sigma(X)$ we write $R(j)$ for the remainder of $j$ divided by $p-3$. We show that the weights $\alpha_{j}^{\prime}=(-1, R(j), R(j)+1)(1 \leq j \leq-\sigma(X))$ satisfy the required property. Recall that, in the assumption of Proposition 2.5, $m, m^{\prime}, r$ and $s$ are nonnegative integers satisfying $m-m^{\prime}=\sigma(X)$ and $3\left(m+m^{\prime}\right)+2(r+s)=\chi(X)$.

Case I If $-3 \sigma(X)+6 \leq \chi(X)$ we take

$$
m=1, \quad m^{\prime}=-\sigma(X)+1, \quad r=\frac{3 \sigma(X)-6+\chi(X)}{2} \quad \text { and } \quad s=0 .
$$

Since we do not require existence of cancelling pairs any choice of $\mathbb{Z}_{p}$-manifolds satisfies the assumption of Proposition 2.5.

Case II If $-3 \sigma(X)+6>\chi(X)$ we take

$$
m=1, \quad m^{\prime}=-\sigma(X)+1, \quad r=0 \quad \text { and } \quad s=\frac{-3 \sigma(X)+6-\chi(X)}{2} .
$$

Our assumption implies $s \geq 0$. We will show the inequality $-\sigma(X)-1 \geq s(\geq 0)$. Then the proof will be completed because Example 2.4 (4) tells that, under the inequality $-\sigma(X)-1 \geq 0$, the number of the cancelling pairs is at least $-\sigma(X)-1$, and hence at least $s$ under the inequality $s \leq-\sigma(X)-1$. Since $-\sigma(X)-1-s=b_{2}^{+}(X)-3$ it suffices to show $b_{2}^{+}(X) \geq 3$. If not, and if $X$ is smooth, Donaldson's Theorems B and $\mathrm{C}$ in [5] imply $b_{2}^{+}(X)=b_{2}^{-}(X) \leq 2$, ie $\sigma(X)=0$ and $\chi(X)=2,4$ or 6 . The case $\chi(X)=6$ is excluded from the assumption $-3 \sigma(X)+6>\chi(X)$ of Case II. The cases $\chi(X)=2$ and 4 are also excluded from our assumption that $X$ is not homeomorphic to $S^{4}$ or $S^{2} \times S^{2}$.

We continue to prove Theorems 1.1 and 1.3.

Fix a prime number $p$ not less than 5. We consider the actions constructed in the proof of Lemma 4.2. So we use the notation there. Choose and fix weights $\alpha_{j}^{\prime}$ for $1 \leq j \leq-\sigma(X)$ as in Lemma 4.2 so that the union of fixed points of the $\mathbb{Z}_{p}$-manifolds $\overline{\mathbb{C} P_{\alpha_{j}^{\prime}}^{2}}$ for $1 \leq j \leq-\sigma(X)$ has $s$ cancelling pairs. We can choose arbitrarily the rest of weights $\alpha_{0}, \alpha_{0}^{\prime}$, and $\beta_{k}$ for $1 \leq k \leq r$. We would like to choose these weights so 
that the inequality of Theorem 4.1 is violated. Since the fixed point data of $S_{\beta}^{4}$ does not contribute to the $\mathbb{Z}_{p}$-index of the Dirac operator on $X$, what we can effectively control are the two weights $\alpha_{0}$ and $\alpha_{0}^{\prime}$.

If we write $I$ for the integer

$$
I=-\sum_{j=1}^{-\sigma(X)} N\left(p, \alpha_{j}^{\prime}\right)-\frac{\sigma(X)}{8} p
$$

then Proposition 3.3 implies

$$
\operatorname{dim}\left(\operatorname{ind}_{\mathbb{Z}_{p}} D_{X}\right)^{\mathbb{Z}_{p}}=I+N\left(p, \alpha_{0}\right)-N\left(p, \alpha_{0}^{\prime}\right) .
$$

On the other hand, a direct calculation shows

$$
\begin{aligned}
& N(p,(-1,0,1))=k \\
& N(p,(-1,1,2))= \begin{cases}l-1 & \text { for } p=4 k \pm 1 \\
l & \text { for } p=12 l \pm 1 \\
l+1 & \text { for } p=12 l+5\end{cases}
\end{aligned}
$$

These imply

$$
N(p,(-1,0,1))-N(p,(-1,1,2))=2 l \quad \text { for } p=12 l+q(q= \pm 1, \pm 5) .
$$

In particular, if we choose $\alpha_{0}=(-1,0,1)$ and $\alpha_{0}^{\prime}=(-1,1,2)$ then we have

$$
\operatorname{dim}\left(\operatorname{ind}_{\mathbb{Z}_{p}} D_{X}\right)^{\mathbb{Z}_{p}}=I+2 l \text { for } p=12 l+q(q= \pm 1, \pm 5),
$$

and if we choose $\alpha_{0}=(-1,1,2)$ and $\alpha_{0}^{\prime}=(-1,0,1)$ then we have

$$
\operatorname{dim}\left(\operatorname{ind}_{\mathbb{Z}_{p}} D_{X}\right)^{\mathbb{Z}_{p}}=I-2 l \text { for } p=12 l+q(q= \pm 1, \pm 5) .
$$

Therefore at least one of the absolute values of the above two is greater than or equal to $2 l$. Hence if $p$ is large enough to satisfy $2 l \geq \max \left\{b_{2}^{+}(X), b_{2}^{-}(X)\right\}$, or the inequality (1-1), then one of the above actions does not satisfy the inequality given in Theorem 4.1. This implies the action is nonsmoothable.

Group actions on 4-manifolds are closely related to cobordisms between 3-manifolds. Interpreting the proofs of Theorems 1.1 and 1.3 in terms of cobordism theory, we obtain the following theorem.

Theorem 4.3 Let $c^{+}$and $c^{-}$be two positive integers satisfying $c^{+} \equiv c^{-} \bmod 8$. If $\left(c^{+}, c^{-}\right)=(2,2)$ or both $c^{+}$and $c^{-}$are greater than 2 , then for any sufficiently large prime number $p$, there is a collection of finitely many lens spaces which satisfies the following three properties. 
(1) All of the lens spaces have fundamental groups $\mathbb{Z}_{p}$.

(2) There exists an oriented spin compact topological 4-manifold $X$ with $b^{+}(X)=$ $c^{+}$and $b^{-}(X)=c^{-}$whose boundary is the disjoint union of the lens spaces.

(3) There dose not exist an oriented spin compact smooth 4-manifold $X$ with $b^{+}(X)=c^{+}$and $b^{-}(X)=c^{-}$whose boundary is the disjoint union of the lens spaces.

Proof We can assume $c^{+} \leq c^{-}$without loss of generality. Let $X$ be a closed, simply connected, spin topological 4-manifold with $b^{+}(X)=c^{+}$and $b^{-}(X)=c^{-}$. Consider the $\mathbb{Z}_{p}$-action on $X$ constructed in the proof of Theorem 1.1 and Theorem 1.3. Note that, since $b^{+}(X)=b^{-}(X)=2$ or both $b^{+}(X)$ and $b^{-}(X)$ are greater than 2 , the construction is valid even if $X$ has no smooth structures. The quotient space $X / \mathbb{Z}_{p}$ is a $V$-manifold whose singularities are several cones on lens space with fundamental group $\mathbb{Z}_{p}$.

Suppose $W$ is a smooth spin $V$-manifold with $b^{+}(W)=c^{+}$and $b^{-}(W)=c^{-}$ whose singularities are the same as those of $X / \mathbb{Z}_{p}$. The index of Dirac operator $D_{W}$ on $W$ is equal to nothing but the right-hand side of the Equation (4-1). Hence, if $p$ is sufficiently large and the weights $\alpha_{0}$ and $\alpha_{j}^{\prime}(0 \leq j \leq-\sigma(X))$ are suitably chosen,

$$
\mid \text { ind } D_{W} \mid \geq \max \left\{c^{+}, c^{-}\right\}
$$

holds as in the proof of Theorem 1.1 and Theorem 1.3. However, it contradicts the inequality

$$
-c^{-}<\text {ind } D_{W}<c^{+}
$$

proved by Fukumoto and Furuta [8].

Remark 4.4 (1) Our construction is not available to find a nonsmoothable $\mathbb{Z}_{5}$ action even if it exists. It is because $N(5, \alpha)=1$ for any weight $\alpha$ and hence $\operatorname{dim}\left(\operatorname{ind}_{\mathbb{Z}_{5}} D_{X}\right)^{\mathbb{Z}_{5}}=3 \sigma(X) / 8$ for any $\mathbb{Z}_{5}$-action on any spin 4 -manifold constructed in Proposition 2.5. This value $3 \sigma(X) / 8$ satisfies the inequality of Theorem 4.1.

(2) Our construction is not available to find a nonsmoothable $\mathbb{Z}_{p}$-action on $S^{2} \times S^{2}$ even if it exists. It is because the fixed point data of any action on $S^{2} \times S^{2}$ constructed in Proposition 2.5 is realized by a smooth action. See Wilczyński [19, Lemma 5.1].

\section{Estimate of $p$}

In the proof of Theorem 1.1 and Theorem 1.3 in the previous section we made use of particular choices of weights. If we use other choices of weights it is likely that 
we could construct nonsmoothable actions for some other prime numbers as well. Theorem 1.4 and Theorem 1.5 are examples of this kind.

Proof of Theorem 1.4 We take $m, m^{\prime}, r$ and $s$ satisfying $n+1=3 m+r, 0 \leq r \leq 2$, $m^{\prime}=m$ and $s=0$. Any choice of $\mathbb{C} P_{\alpha_{i}}^{2}(1 \leq i \leq m), \overline{\mathbb{C} P_{\alpha_{j}^{\prime}}^{2}}(1 \leq j \leq m)$, and $S_{\beta_{k}}^{4}$ $(1 \leq k \leq r)$ satisfies the assumption of Proposition 2.5.

Take $\alpha_{i}=(-1,0,1)$ for every $1 \leq i \leq m$ and $\alpha_{j}^{\prime}=(-1,1,2)$ for every $1 \leq j \leq m$. If this action is smooth with respect to some smooth structure on $X$ then

$$
\begin{aligned}
\operatorname{dim}\left(\operatorname{ind}_{\mathbb{Z}_{p}} D_{X}\right)^{\mathbb{Z}_{p}} & =m N(p,(-1,0,1))-m N(p,(-1,1,2)) \\
& =2 l m \quad \text { for } p=12 l+q(q= \pm 1, \pm 5)
\end{aligned}
$$

by Proposition 3.3 and the Equation (4-2). Hence, for any $n$ and $p$ satisfying

$$
2 l m \geq n=3 m+r-1 \text { for } p=12 l+q(q= \pm 1, \pm 5),
$$

the action does not satisfy the inequality of Theorem 4.1 .

Proof of Theorem 1.5 Let $p$ be 11 and $X$ the topological manifold homeomorphic to $K 3$ surface.

For $\alpha_{1}^{\prime}=(-1,1,2), \alpha_{2}^{\prime}=(-1,2,3)$ and $\alpha_{3}^{\prime}=(-1,3,4)$ the $\mathbb{Z}_{p}-$ manifolds $\overline{\mathbb{C} P_{\alpha_{1}^{\prime}}^{2}}$, $\overline{\mathbb{C} P_{\alpha_{2}^{\prime}}^{2}}$ and $\overline{\mathbb{C} P_{\alpha_{3}^{\prime}}^{2}}$ have two cancelling pairs as in Example $2.4(2)$. On the other hand, for $\alpha_{4}^{\prime}=(-2,2,4)$ the pair consisting of $[1,0,0]$ on $\overline{\mathbb{C} P_{\alpha_{3}^{\prime}}^{2}}$ and $[1,0,0]$ on $\overline{\mathbb{C} P_{\alpha_{4}^{\prime}}^{2}}$ is a cancelling pair. So by taking weights $\alpha_{j}^{\prime}$ for $1 \leq j \leq 16$ as

$$
\alpha_{j}^{\prime}=\alpha_{k}^{\prime} \quad \text { for } j \equiv k \bmod 4 \text { and } 1 \leq k \leq 4 \text {. }
$$

Sixteen $\mathbb{Z}_{p}$-manifolds $\overline{\mathbb{C} P_{\alpha_{j}^{\prime}}^{2}}(1 \leq j \leq 16)$ have 12 cancelling pairs, that is, they satisfy the assumption of Proposition 2.5 for $m=r=0, m^{\prime}=16$ and $s=12$.

Since

$$
N(11,(-1,1,2))=N(11,(-1,2,3))=N(11,(-1,3,4))=N(11,(-2,2,4))=1 \text {, }
$$

if the above action is smooth with respect to some smooth structure of $X$ then

$$
\operatorname{dim}\left(\operatorname{ind}_{\mathbb{Z}_{11}} D\right)^{\mathbb{Z}_{11}}=6
$$

by Proposition 3.3. The action therefore does not satisfy the inequality of Theorem 4.1 because $b_{2}^{+}(X)$ is equal to 3 . This implies the action is nonsmoothable.

Corollary 5.1 We have $11 \in \operatorname{NS}\left(K 3 \#\left(\#^{t} S^{2} \times S^{2}\right)\right)$ for $t=1,2$ and 3 . 
Proof Let $X$ be $K 3 \#\left(\#^{t} S^{2} \times S^{2}\right)$ for $t=\underline{1,2}$, or 3 . Choose weights $\beta_{k}$ for $1 \leq k \leq t$ arbitrarily, then the $\mathbb{Z}_{p}$-manifolds $\overline{\mathbb{C} P_{\alpha_{j}^{\prime}}^{2}}$ for $1 \leq j \leq 16$ in the proof of Theorem 1.5 and $S_{\beta_{k}}^{4}$ for $1 \leq k \leq t$ satisfy the assumption of Proposition 2.5 for $X$. If the action is smoothable

$$
\operatorname{dim}\left(\operatorname{ind}_{\mathbb{Z}_{11}} D_{X}\right)^{\mathbb{Z}_{11}}=6
$$

since the fixed points from $S_{\beta_{k}}^{4}$ does not contribute. Then the inequality of Theorem 4.1 is not satisfied, which implies that the action is nonsmoothable.

Remark 5.2 In the case of $n=2$ or 3, the estimate of $p$ in Theorem 1.4 coincides with those in Theorem 1.1 and it is not an improvement. In the case of $n \geq 4$, Theorem 1.4 gives an improvement. Still better estimations might be obtained using the construction in Section 2 using other choices of $m, m^{\prime}, r$ and $s$.

\section{References}

[1] J Bryan, Seiberg-Witten theory and $\mathbf{Z} / 2^{p}$ actions on spin 4-manifolds, Math. Res. Lett. 5 (1998) 165-183 MR1617929

[2] W Chen, S Kwasik, Symmetries and exotic smooth structures on a K3 surface, J. Topol. 1 (2008) 923-962 MR2461861

[3] P Dolbeault, Sur la cohomologie des variétés analytiques complexes, C. R. Acad. Sci. Paris 236 (1953) 175-177 MR0052771

[4] S K Donaldson, An application of gauge theory to four-dimensional topology, J. Differential Geom. 18 (1983) 279-315 MR710056

[5] S K Donaldson, Connections, cohomology and the intersection forms of 4-manifolds, J. Differential Geom. 24 (1986) 275-341 MR868974

[6] A L Edmonds, Construction of group actions on four-manifolds, Trans. Amer. Math. Soc. 299 (1987) 155-170 MR869405

[7] A L Edmonds, J H Ewing, Realizing forms and fixed point data in dimension four, Amer. J. Math. 114 (1992) 1103-1126 MR1183533

[8] Y Fukumoto, M Furuta, Homology 3-spheres bounding acyclic 4-manifolds, Math. Res. Lett. 7 (2000) 757-766 MR1809299

[9] I Hambleton, R Lee, Smooth group actions on definite 4-manifolds and moduli spaces, Duke Math. J. 78 (1995) 715-732 MR1334207

[10] I Hambleton, M Tanase, Permutations, isotropy and smooth cyclic group actions on definite 4-manifolds, Geom. Topol. 8 (2004) 475-509 MR2033485 
[11] M Klemm, Finite group actions on smooth 4-manifolds with indefinite intersection form, $\mathrm{PhD}$ thesis, McMaster University (1995) Available at http:// digitalcommons.mcmaster.ca/opendissertations/2369

[12] K Kodaira, On a differential-geometric method in the theory of analytic stacks, Proc. Nat. Acad. Sci. U. S. A. 39 (1953) 1268-1273 MR0066693

[13] S Kwasik, T Lawson, Nonsmoothable $Z_{p}$ actions on contractible 4-manifolds, J. Reine Angew. Math. 437 (1993) 29-54 MR1212252

[14] S Kwasik, K B Lee, Locally linear actions on 3-manifolds, Math. Proc. Cambridge Philos. Soc. 104 (1988) 253-260 MR948910

[15] X Liu, N Nakamura, Pseudofree $\mathbb{Z} / 3$-actions on $K 3$ surfaces, Proc. Amer. Math. Soc. 135 (2007) 903-910 MR2262889

[16] X Liu, N Nakamura, Nonsmoothable group actions on elliptic surfaces, Topology Appl. 155 (2008) 946-964 MR2401205

[17] N Nakamura, Bauer-Furuta invariants under $\mathbb{Z}_{2}$-actions, Math. Z. 262 (2009) 219233 MR2491607

[18] D M Wilczyński, Periodic maps on simply connected four-manifolds, Topology 30 (1991) 55-65 MR1081933

[19] D M Wilczyński, On the topological classification of pseudofree group actions on 4-manifolds. I, Math. Z. 217 (1994) 335-366 MR1306665

Graduate School of Mathematical Sciences, The University of Tokyo 3-8-1 Komaba Meguro-ku, Tokyo 153-8914, Japan

nkiyono@mail.ecc.u-tokyo.ac.jp

Received: 26 April 2010 Revised: 22 February 2011 